\title{
2011 Acknowledgements
}

The Editors of the World Journal of Surgery wish to extend our appreciation and gratitude to all who have provided editorial assistance to the WJS in 2011.

The names of all guest reviewers can be found at:

www.springer.com/268

Thank you for your generous support!

(C) Société Internationale de Chirurgie 2011 